\title{
Eastern Medicine Approaches to Male Infertility
}

\author{
Min Hu, M.D. ${ }^{1}$, Yuehui Zhang, M.D., Ph.D. ${ }^{1}$, Hongli Ma, M.D. ${ }^{1}$, \\ Ernest HY Ng, M.D., Ph.D. ${ }^{2}$, Xiao-Ke Wu*, M.D., Ph.D. ${ }^{1}$ \\ ${ }^{1}$ Department of Obstetrics and Gynecology, Key Laboratory and Unit of Infertility in Chinese Medicine, \\ First Affiliated Hospital, Heilongjiang University of Chinese Medicine, Harbin, P.R. CHINA \\ ${ }^{2}$ Department of Obstetrics and Gynecology, University of Hong Kong, Queen Mary Hospital, Hong Kong
}

Address for correspondence and reprint requests: $\quad$ Xiao-Ke $\mathrm{Wu}$

Department of Obs \& Gyn, First Affiliated Hospital, Heilongjiang University of Chinese Medicine

Harbin 150040, P.R. CHINA Tel \& Fax: +86-451-8213 $0094 \quad$ Mobil: +86-137 96025599

E-mail: xiaokewu2002@vip.sina.com

Abstract: Male factor is a common cause of infertility and the male partner must be systematically evaluated in the workup of every infertile couple. Various eastern medical strategies have been tried with variable success. This article describes the clinical effects of eastern medicine approaches including acupuncture, Chinese herbal medicine, massage, yoga, tai chi and qi gong which could improve the sperm parameters and motility, genital inflammatory, as well as immune system disorder, sexual dysfunction, and varicocele. Acupuncture reduces inflammation, increases sperm motility and semen parameters, modulates immune system, as well as improves sexual and ejaculatory dysfunction in male infertility. The clinical effects may mediate via activation of somatic afferent nerves innervating the skin and muscle. Chinese herbal medicines may also exert helpful effects in male infertility, and it is worth noting that some herbal drugs may result in male infertility. Massage also exerts positive effects in male infertility. Nevertheless, the mechanisms of clinical effects are unclear. Tai chi, qi gong and yoga have not been investigated in male infertility, but it has been reported to regulate endocrine and central or autonomic nervous systems. In conclusion, eastern medical approaches have beneficial on reproductive effects in male infertility. However, future well-designed randomized clinical control trials are needed to evaluate the safety, efficacy, and mechanisms of eastern medical approaches for male infertility.

Keywords: Male Infertility; Acupuncture; Chinese Herbal Medicine; Massage; Yoga; Tai Chi; Qi Gong 
Infertility is a common clinical problem affecting $5 \%$ to $30 \%$ of couples worldwide, with higher rates in developing countries (1). Male factors may be responsible for $40-60 \%$ of cases (2). Interference in any physiological steps of spermatogenesis or ejaculation may lead to male infertility As such, the male partner must be systematically evaluated in the investigation of an infertile couple. Treatment of male factor infertility can involve targeted therapy, or it can be just empirical using medical therapy or assisted conception techniques for patients in whom no underlying cause has been identified.

The present study characterizes of eastern medicine among a cohort of couples seeking fertility care were prospectively, such as Chinese herbal medicine, acupuncture, massage, and yoga. Some infertile couples may turn to eastern medical approaches in an attempt to become pregnant as these treatments may be perceived as being lower cost, safer, or more effective (3-6). In a clinical study, 428 couples present for evaluation of infertility (7). After 18 months of observation, $22 \%$ of the couples had tried acupuncture for treatment of infertility, $17 \%$ herbal therapy, $5 \%$ a form of body work, and $1 \%$ had utilized meditation. Various eastern medical strategies could be an adjunct to conventional medical management of male infertility.

This article describes how eastern medicine approaches could improve the symptoms and manage the primary disease of male infertility, such as genital inflammatory, immune system disorder, abnormal semen parameters and sexual dysfunction, as well as varicocele.

\section{Acupuncture}

Acupuncture is an ancient method that has been used for centuries in the treatment and prevention of many diseases. The Chinese have provided an ancient explanation of traditional acupuncture based on the principle of energy flow around the body in channels called "meridians". Inserting acupuncture needles or stimulating acupoints using acupressure can reestablish harmony. It is gaining widespread popularity in modern medicine as a complementary treatment for fertility and as a supportive intervention in in vitro fertilization (IVF).

Acupuncture has been documented in several studies as having a positive effect on sperm parameters. It increased total functional sperm fraction, percentage of viability, total motile sperm per ejaculation, and overall integrity of the axoneme(8). Several clinical trials have investigated the use of acupuncture as a therapy for the primary diseases of male infertility, which mainly include sexual dysfunction, immune system disorder, idiopathic infertility, genital inflammation as well as varicocele. Nowadays, more and more clinical and animal researches have been conducted to evaluate the efficacy of acupuncture for male infertility and explore its mechanisms. 


\subsection{Clinical Efficacy of Acupuncture in Male Infertility}

Male sexual dysfunction Sexual dysfunction is prevalent in men. Although new pharmaceutical agents have been identified for male erectile problems, sexual desire and orgasm disorders, individuals with sexual dysfunction often seek alternative therapies, including acupuncture (9). Sunay et al.(10) recruited 90 patients with premature ejaculation were randomized into paroxetine, acupuncture, and placebo groups for 4 weeks. The Premature Ejaculation Diagnostic Tool (PEDT) scores after treatment were significantly lower in the paroxetine and acupuncture groups. Although acupuncture was less effective than paroxetine in delaying ejaculation, acupuncture was significantly better than placebo.

Successful sexual functioning is a complex process involving psychological and social responses as well as neurological, biochemical and vascular processes. A pilot study indicated that acupuncture can be an effective treatment option in patients with psychogenic erectile dysfunction (11). Twenty patients following a varying number of acupuncture sessions treatment demonstrated successful erections(12). Although there is some encouraging evidence that acupuncture is an effective intervention in sexual dysfunction and erectile dysfunction $(13,14)$, the evidence is insufficient and further research is required to establish the value of acupuncture in the treatment of sexual dysfunction in men.

Abnormal semen parameters Spermatogenesis is a complex process controlled by a network of endocrine and other regulatory factors. Abnormal semen parameters are found in $50 \%$ of males undergoing infertility evaluation with the most common finding being oligoastenoteratozoospermia (15). The effect of acupuncture on male infertility, especially on the idiopathic oligospermia, asthenozoospermia and azoospermia, is still a matter of debate. There is some evidence of improvement in sperm quality when acupuncture is performed on males with abnormal semen parameters, and the incidence of side effects is low (16-18). Zhang et al.(19) investigated 22 men with abnormal semen parameters who failed in intracytoplasmic sperm injection (ICSI) were treated with acupuncture twice weekly for 8 weeks, followed by ICSI treatment again. There was no significant difference in sperm concentration and sperm motility between before and after acupuncture. After acupuncture, quick sperm motility, normal sperm ratio and the fertilization rates were significantly higher than those before acupuncture. The embryo quality after acupuncture was also improved. Bidouee et al.(20) treated a case of an azoospermic male in whom spermatogenesis occurred and he had sperm in his ejaculate after two courses of acupuncture. This is the first report of successful treatment of idiopathic non-obstructive azoospermia with acupuncture in Iran. Otherwise, Pei et al.(21) showed a statistically significant improvement in acrosome position and shape, 
nuclear shape, axonemal pattern and shape, accessory fibers of sperm organelles, and the ultrastructural integrity of spermatozoa in men with abnormal semen parameters after acupuncture compared to untreated control groups. Acupuncture treatments were administered twice a week for 5 weeks (25 minutes per session). Very recently, Dieterle et al.(18) reported the first prospective randomized placebo-controlled study on the effect of acupuncture in subfertile males. Fifty-seven subfertile patients with severe oligoasthenozoospermia were randomly allocated into either acupuncture $(n=28)$ or placebo acupuncture groups $(n=29)$. Both acupuncture and placebo acupuncture treatments were applied for 45 minutes, twice a week for 6 weeks. A significant increase in the percentage of total motile sperm was found after acupuncture treatment.

It is a usual practice to combine acupuncture and moxibustion in patients with specific syndrome differentiation in order to enhance the efficacy of acupuncture treatment in China. The use of both acupuncture and moxibustion in subfertile men had also been reported in previous studies(22,23). A randomized controlled trial about the effect of acupuncture plus clomiphene on 108 males with idiopathic normal gonadotrophic oligospermia ( $<20$ million/mL) was reported by He Xinyun(23). In the acupuncture plus clomiphene group, acupuncture treatments with the addition of electrical stimulation or moxibustion to specific acupoints were administered every other day for 3 months. The control group received clomiphene only. It revealed that a significantly higher percentage of achieving pregnancy or normalization of semen parameters was found in acupuncture and clomiphene combination therapy (74\%) when compared with clomiphene alone (52\%). Gurfinkel et al.(24) randomized 19 men with semen abnormalities into study ( $\mathrm{n=9}$ ) and control groups $(n=10)$. Patients in the study group received 25 -minute classic acupuncture followed by a 20-minute moxibustion twice a week for 10 weeks. Those in control group received acupuncture and moxibustion at non-therapeutic indifferent points. This study revealed the ratio of the normal form sperm increased significantly after classic acupuncture and moxibustion.

However, the underlying mechanisms how acupuncture improves semen parameters remain unknown. Using a scrotal heat-treated rat model to study the effect of acupuncture in spermatogenesis, it has been shown that acupuncture enhanced germ cell proliferation through improvement of Sertoli cell functions. This may facilitate the recovery of spermatogenesis and may restore normal semen parameters in subfertile patients (25).

Immunological infertility Acupuncture can achieve the effects of adjusting and enhancing immunity system and have a bidirectional modulation of the specific and non-specific immunity of the body(26). Fu (27) 
and Lun et al.(28) investigated the effects of acupuncture on male immune infertility. One hundred 100 males with positive antisperm antibody (AsAb) were randomly divided into acupuncture, acupuncture combined with herbal drugs and oral prednisone. The results showed that sperm density and serum positive AsAb level in the use of acupuncture group were significantly superior to those in the control group. The positive rate of $A s A b$ in all groups decreased, but the negative-turning rate of AsAb in the acupuncture-drug group was more obvious. Many researches focus on mechanisms of acupuncture in treatment of male immune infertility from clinical trials (29). Finally, it was suggested that the mechanism of the electroacupuncture in treatment of male immune infertility is possibly related with regulation of the contents of nitric oxide and trace elements such as $\mathrm{Zn}, \mathrm{Cu}$ and Fe. Indeed, laboratory research has confirmed the systemic immunoregulatory actions of acupuncture (30). It can also act on the nerve-endocrine-immunity network, improve the internal environment of reproduction, eliminate the exogenous allergenic, and produce antigen reaction and anti-allergic reaction.

Genital inflammation Poor spermatogenesis in patients with inflammation of the genital tract is associated with scrotal hyperthermia, which is another impotent reason leading infertility. Acupuncture may be a useful, nontraumatic treatment for males with very poor sperm density, especially those with a history of genital tract inflammation (31). A pilot trial included 39 men who were referred for acupuncture owing to low sperm count related to high scrotal skin temperature (17). In accordance with the principles of traditional acupuncture and syndrome diagnosis, acupuncture points appropriate for the deficiency of the kidneys (hormonal imbalance) and damp-heat syndromes (inflammation of the genital tract) were selected. Following acupuncture treatment, sperm count was increased in 15 out of these 17 patients.

Moreover, male accessory gland infection has been linked to male infertility, and acupuncture therapy exhibited a definite effect in the treatment of chronic prostatitis. In a systematic review, thirteen eligible reports were identified, including 861 cases and 738 controls. The effectiveness and cure rates were significantly higher in the acupuncture therapy group $(32,33)$. Many clinical trials have confirmed that acupuncture or electroacupuncture could improve chronic prostatitis symptoms, such as the pain, voiding symptoms, quality of life, and NIH CPSI total scores (34-37). The analgesic effect of electroacupuncture was confirmed by observing prostaglandin E2 in the postmassage urine, which decreased significantly from $98.18 \pm 19.36 \mathrm{pg} / \mathrm{ml}$ to $71.43 \pm 14.98 \mathrm{pg} / \mathrm{ml}$ (37). And no adverse event that was related to acupuncture was reported in any patient throughout the treatment period (38).

Varicocele Varicocele can be found in about $35 \%$ of male infertility (39). It leads to stagnation in the 
testicular microcirculation and hypoxic-ischemic degenerative changes in all cellular types at the sperm production site. One would expect that acupuncture would interrupt the perioxidation process and thereby improve sperm output in patients with varicocele (40). It is reported that testicular arterial blood flow is significantly decreased in men with varicocele (41). The testis, as a homologous organ of the ovary, may also respond to abdominal electroacupuncture with an increase of testicular artery. There is a prospective, randomized study of electroacupuncture to clarify the role of the abdominal acupuncture points and the frequency of short-term electroacupuncture stimulation on testicular blood flow in humans (42). This article was for the first time to demonstrate location- and frequency-specific testicular artery responses to abdominal electroacupuncture in humans. Single-use 0.20 by $13 \mathrm{~mm}$ sterile stainless steel acupuncture needles were used for acupuncture needling in eighty healthy male volunteers. The needles were inserted relatively superficially $(\sim 1 \mathrm{~cm})$, sufficiently deep to stimulate sensory elements of the skin while remaining minimally invasive for patients. It is showed that the $10-\mathrm{Hz}$ electroacupuncture stimulation of ST29 (guilai) increased testicular blood flow. Further investigation is required to ascertain whether these findings may be helpful in the clinical treatment of infertile men.

\subsection{Physiological Mechanisms of Acupuncture in Male Infertility}

The primary mechanism for acupuncture's clinical effects is activation of somatic afferent nerves innervating the skin and muscles, which may modulate somatic, autonomic nervous system activity, endocrine and metabolic functions. Acupuncture can be seen as having effects through the nervous system in the broad categories of local, segmental, extrasegmental, and central effects, or direct mechanical effects on infertility (43). Experimental studies suggest that acupuncture effects are mediated by changes in activity of the autonomic nervous system and stimulation of neuropeptides/ neurotransmitters which may be involved in the pathogenesis of infertility (16). At the end of acupuncture treatment on chronic pelvic pain, the result is $\beta$-endorphin and leucine-enkephalin levels were both higher. These effects of acupuncture could be explained by the following hypothetical mechanisms: local peripheral mechanisms, spinal (segmental) mechanisms, and supraspinal (extrasegmental or central) mechanisms $(16,44,45)$. The location, regional anatomy and innervation of acupoints commonly used on male infertility described in Table 1.

Acupuncture could relieve edema and inflammatory infiltration, as well as reduce local inflammatory reactions(46). Meanwhile, most of the acupuncture studies focused on immunological regulation and microcirculation. Yuan et al.(47) explored the effect of acupuncture on patients with chronic pelvic pain syndromes, and its therapeutic mechanism. Forty-seven cases were treated with electroacupuncture on CV3 
(Zhongji), ST29 (Guilai), SP9 (Yinlingquan), SP6 (Sanyinjiao), CV4 (Guanyuan), ST28 (Shuidao), SP10 (Xuehai) and LR3 (Taichong) as main acupoints. The levels of interleukin-8 (IL-8), interleukin-10 (IL-10) and tumor necrosis factor-alpha (TNF- $\alpha$ ) in prostate fluid were reduced after acupuncture treatment. Acupuncture may be effective in male infertility disease through reducing the levels of IL-8, IL-10 and TNF- $\alpha$ to relieve inflammatory reaction. In addition, acupuncture could increase the content of secreted immunoglobulin A in the prostatic fluid and rectify the local immune function of the prostate (48). T lymphocyte subgroup has the favorable changes in the peripheral blood of male immune infertility after acupuncture (49). The mechanism may also be related to the increase of acid phosphatase (ACP) content in seminal plasma for male immune infertility by acupuncture (50). These data indicate that acupuncture could regulate the endocrine function and rectify the abnormal immune function.

Table 1. The location, regional anatomy and innervation of acupoints commonly used on male infertility.

\begin{tabular}{|c|c|c|c|c|}
\hline Acupoint name & Location & Muscle & Innervation & Common therapy of male infertility \\
\hline CV3(Zhongji) & 4 cun caudal to the umbilicus & Fibrous tissue, linea alba & L1 & $\begin{array}{l}\text { Chronic postatitis, Impotence, } \\
\text { Varicocele, Azoospermia, } \\
\text { Oligoasthenozoospermia, }\end{array}$ \\
\hline CV4(Guanyuan) & $\begin{array}{l}\text { At } 3 \text { cun below the center of the umbilicus on } \\
\text { the lower abdomen and on the anterior } \\
\text { midline }\end{array}$ & Fibrous tissue, linea alba & Th12 & $\begin{array}{l}\text { Chronic postatitis, Impotence, } \\
\text { Immune infertility, Azoospermia, } \\
\text { Oligoasthenozoospermia, }\end{array}$ \\
\hline SP6(Sanyinjiao) & At 3 cun proximal to the medial malleolus & $\begin{array}{l}\text { Mm. flexor digitorum longus, } \\
\text { tibialis posterior }\end{array}$ & L4-5, S1-2 & Ejaculation disorders \\
\hline SP9(Yinlingquan) & Below medial tibia chondyle & M. gastrocnemius & S1-2 & Chronic postatitis, Azoospermia \\
\hline ST29(Guilai) & $\begin{array}{l}1 \text { cun cranial to the pubic bone and } 2 \text { cun } \\
\text { lateral to the midline }\end{array}$ & $\mathrm{M}$ rectus abdominis & Th6-12 & Oligoasthenozoospermia, Varicocele \\
\hline BL23(Shenshu) & $\begin{array}{l}\text { Under the } 2 \text { nd spinous process of lumbar } \\
\text { vertebra, next to } 1.5 \text { cun }\end{array}$ & Erector spinae & L1 & $\begin{array}{l}\text { Chronic postatitis, Impotence, } \\
\text { Immune infertility, Azoospermia, } \\
\text { Oligoasthenozoospermia }\end{array}$ \\
\hline BL32(Ciliao) & $\begin{array}{l}\text { At the } 2 \text { nd posterior sacral foramen on the } \\
\text { sacrum; and the posterior ramus of the S2 } \\
\text { nerve }\end{array}$ & Erector spinae & L2-S4 & $\begin{array}{l}\text { Chronic postatitis, Immune infertility, } \\
\text { Oligoasthenozoospermia, Ejaculation } \\
\text { disorders }\end{array}$ \\
\hline LR3(Taichong) & $\begin{array}{l}\text { Between metatarsale I \& } \Pi \text {, just distal to } \\
\text { the caput }\end{array}$ & M. Interosseous dorsalis I & S2-3 & $\begin{array}{l}\text { Chronic postatitis, Azoospermia, } \\
\text { Oligoasthenozoospermia, impotence, } \\
\text { Ejaculation disorders }\end{array}$ \\
\hline PC6(Neiguan) & $\begin{array}{l}2 \text { cun proximal to processus styloideus radii, } \\
\text { between the tendons of palmaris longus and } \\
\text { flexor carpi radialis }\end{array}$ & $\begin{array}{l}\text { M. flexor digitorum } \\
\text { superficilais }\end{array}$ & C8, Th1 & Immune infertility, \\
\hline GV 20(Baihui) & $\begin{array}{l}\text { On the top of the head, } 5 \text { cun directly above } \\
\text { the posterior hairline, approximately on the } \\
\text { midpoint of the line connecting the apexes of } \\
\text { the two auricles }\end{array}$ & Aponeurosis epicranii & $\begin{array}{l}\mathrm{C} 2-3, \mathrm{~N} \text {. } \\
\text { trigeminus }\end{array}$ & $\begin{array}{l}\text { Immune infertility, Azoospermia, } \\
\text { Impotence }\end{array}$ \\
\hline
\end{tabular}




\section{Chinese Herbal Medicine}

Chinese herbal medicine (CHM) is an integral part of traditional Chinese medicine (TCM), which assesses the cause of the disease and gives treatment based on the differentiation types. Because of the complex of etiology, Chinese physicians have different views on the etiology and pathology of male infertility resulting in different differentiation types. Most TCM doctors believe that male infertility is closely related to the disorders of the spleen, liver and especially the kidney. In the TCM theory, the kidney is the organ which stores kidney-essence and plays a crucial role in reproduction. In western medicine, male infertility usually is caused by various reasons such as abnormal semen parameters, immunological cause, erectile dysfunction and chronic prostatitis, the differentiation types of male infertility in TCM are various. CHM treatment typically consists of prescriptions of a combination of herbs in different proportions. The prescriptions are based on four main diagnostic procedures: observation, listening, questioning, and pulse analysis(51). The comprehensive analysis of clinical information gained by the TCM doctor is used to guide the syndrome differentiation and treatment by $\mathrm{CHM}$ formulae. We will discuss the clinical treatment effects about different etiology of male infertility.

\subsection{Clinical Treatment of Male Infertility}

\section{Abnormal semen parameters}

Furuya et al.(52) used Bu-zhong-yi-qi-tang (Hochu-ekki-to) in 22 men with abnormal semen parameters for more than three months without adverse effect. The sperm concentration and motility of 22 patients in pre-treatment were $\left(28 \pm 31 \times 10^{6} / \mathrm{ml}\right)$ and $(32 \pm 17 \%)$, respectively. Sperm concentration $\left(42 \pm 38 \times 10^{6} / \mathrm{ml}\right)$ and motility (39 $\pm 19 \%)$ were significantly increased 3 months after the herb administration. Interestingly, an inverse correlation between seminal plasma IL-6 level and sperm concentration was found before treatment. So there was no significantly change on seminal plasma IL-6 and IL-8 after the treatment. But after the administration of herb, seminal plasma soluble Fas (sFas) levels significantly increased and were correlated with sperm concentration. It is possible that certain kinds of cytokine in the seminal plasma might play some role in improving semen quality after herb administration.

Bu-zhong-yiqi-tang (Hochu-ekki-to) is a Chinese herbal medicine that has been used for abnormal semen parameters of unknown cause with some favorable clinical results. However, the mechanisms of this medicine on male infertility are not known. Amanor $\mathrm{T}$ et al.(53) investigated the in vitro effects of Hochu-ekki-to on sperm motility of human semen. Sixteen semen samples were obtained from 14 normal volunteers. They found that the motility of sperm in 10 and $100 \mu \mathrm{g} / \mathrm{mL}$ of Hochu-ekki-to solution for 1-6 h 
was significantly higher than that of the control groups. The motility of semen with $1 \mu \mathrm{g} / \mathrm{mL}$ was also significantly higher than that of the control group after $4 \mathrm{~h}$ incubation.

Immunological infertility Immunological infertility in men refers to the presence of anti-sperm antibodies in the serum or seminal fluid. This condition should be treated on the principle of strengthening the body resistance and dispelling the pathogenic factors. Its differentiation type in the clinic belongs to kidney-yin deficiency and damp-heat according to TCM theory. Sun et al.(54) observed the YiKang decoction has a stable effect for male infertility than prednisone. They treated 100 cases of male infertility with YiKang decoction, while 100 cases with prednisone as the controls. After the treatment for 3 months, the serum and seminal plasma AsAb levels decreased significantly, while the sperm density and percentage of motile spermatozoa increased significantly in both the treatment group and the control group. These changes in treatment group were more significant. Another controlled trial selected 90 men with immunological infertility who were randomly divided into two groups(55). Sixty men were treated by Hu-zhang-dan-shen-yin in the treatment group for 3 months while another 30 men in the control group was treated by prednisone for 3 months. The total levels of antisperm antibody declined significantly in the treatment group than that of controls. The clinical manifestations and symptoms such as backache, pain in knees, lumbar debility, sagging distention of the perineum and testis were more alleviated in the treatment group than those in the control after the treatment. A case-control study of 90 men with immunological infertility found that the total effective rate in the control group with prednisone and clomiphene for 2 months was $56.6 \%$, while the corresponding rate in the treatment group was $80.0 \%$ with the decoction Sheng-Jing-Zhong-Zi-Tang everyday for 60 days(56).

Chronic genital infection The infection in accessory gland including chronic prostatitis, vesiculitis, epididymitis etc. Its differentiation types belong to damp-heat and blood stasis according to TCM theory. Jia et al. (57) applied suppository consisting of several kinds of Chinese herbal medicine to 104 patients with chronic prostatitis. Of the 104 cases, 24 cases were short-term cured, 38 men markedly improved, 26 improved, and 16 cases were ineffective, with a total effective rate of $84.6 \%$. Sun et al. (58) conducted a randomized controlled trial and divided 60 cases into treatment and control groups in a ratio of 1:1. The cure rate was $26.17 \%$, the total effective rate was $86.17 \%$ in the decoction group, while in the control group treated with antibiotic plus vitamin $\mathrm{E}$ the corresponding rates were $6.17 \%$ and $76.17 \%$, respectively. There are some external therapies of $\mathrm{CHM}$ for chronic prostatitis in addition to traditional treatment of oral $\mathrm{CHM}$ decoction(59). The herbal drugs can be made into suppository, bolus or troche to be put into the rectum. The herbal drugs can also be grinded into powder mixing with plaster or other excipients to apply on the skin for 
the local absorption. Some other doctors applied retention-enema therapy of CHM decoction.

\subsection{Safety and Efficacy of CHMs in Male Infertility}

Unfortunately, there are very few RCTs on CHM for male infertility and moreover they are very small in number (60). Most of the studies are case-control reports or just case reports. In the few RCT studies, they have poor methodological quality and only one article mentioned the randomization method employed in their study. Most, not surprisingly, have been conducted mainly in Chinese populations and published in Chinese and thus are not easily known to everyone. It is important that transparent, pragmatic but rigorous clinical research methodologies are developed that accommodate the complex, individualised, and changing nature of $\mathrm{CHM}$ interventions.

So far, no study has reported any adverse events after taking CHM for male infertility. But this does not mean that TCM is very safe. Several studies have indicated that some herbal drugs may result in male infertility. Tripterygium hypoglaucum Hutch $(\mathrm{TH})$ is a perennial used in TCM for the treatment of rheumatoid arthritis and various skin disorders. One study showed that daily oral doses of TH significantly reduced the fertility of male rats without apparent toxicity (61). The sperm concentration, motility and motility grade of 13 men taking $\mathrm{TH}$ were compared to those of 11 untreated controls. It was concluded that TH therapy did not affect testosterone, FSH, LH levels, and its antifertility effects appeared to be reversible (62). Matlin et al.(63) revealed the antifertility agents extracts from tripterygium wilfordh hook F. were the diterpenes triptolide and tripdiolide and an isomer of tripdiolide.

\subsection{The Mechanisms of CHMs in Male Infertility}

Currently, the physiological mechanisms for most CHMs in male infertility are unknown. Table 2 lists several CHMs used in male infertility and their proposed mechanism of action. Over the twenty years substantial pharmacological research has been performed to investigate the chemical constituents of a variety of $\mathrm{CHMs}$, many of the $\mathrm{CHMs}$ have strong antioxidant activity (64) and function like gonadotropins which can increase the production of testosterone and improved sperm motility.

A clinical research found that Wu-Zi-Yan-Zong-Wan (65) have protective effect on oxidative damage of mtDNA in aged men, it could reduce the mtDNA deletions and raise the activities of mitochondrial respiratory chain complexes I, IV in periphery blood leukocytes of aged men. CHM Fu Pen Zi (Palmleaf Raspberry Fruit) can increase testosterone levels of rats but reduce estradiol levels (66). While leydig TM3 mouse cells cultured with herb $\mathrm{Ba} \mathrm{Ji}$ Tian (Radix moridae officinalis) have been shown to increase the production of testosterone having protective effects against hydrogen peroxide-induced oxidative stress(67). 
Peng SJ (68) found the sperm motility had been markedly improved after incubation with herb Tu Si Zi (Semen cuscutae). The herb possessed strong antioxidant activity which played a role in stabilizing the sperm membrane and prevent/reduce lipid peroxidation of the sperm plasma membrane that may result in sperm dysfunction and cell death(69). The main chemical composition of herb Cuscuta seed and Epimedium is flavonoids which has important effects on the hypothalamic-pituitary-gonadal axis endocrine function(70). Another herb Huang Qi (Mongolian Milkvetch Root) was capable of preventing cerebral oxidative injury in rats in-vivo(71). There are some other CHMs for male infertility which may increase trace elements and treatment for infection $(72,73)$.

Table 2. Partial list of CHMs used to treat male infertility and their proposed effects.

\begin{tabular}{|c|c|c|c|}
\hline Effect & Chinese Name & Latin Name & English Name \\
\hline \multirow[t]{4}{*}{ Increase the production of testosterone } & Ba Ji Tian & Radix moridae officinalis & Morinda root \\
\hline & Rou Cong Rong & Herba Cistanches Deserticolae & Desertliving Cistanche Herb \\
\hline & Fu Pen Zi & Fructus Rubi Chingii & Palmleaf Raspberry Fruit \\
\hline & Yin Yang Huo & Herba epimedii & Epimedium \\
\hline \multirow[t]{2}{*}{ Improve Sperm motility } & Tu Si Zi & Semen cuscutae & Dadder seed/ cuscuta seed \\
\hline & Gou Qi Zi & Fructus lycii chinensis & Lycium fruit \\
\hline Strong antioxidant activity & Huang Qi & Radix Astragali Mongolici & Mongolian Milkvetch Root \\
\hline Enhancing immune function & Huang Jing & Rhizoma Polygonati Sibirici & Siberian Solomonseal Rhizome \\
\hline \multirow[t]{2}{*}{ Treat the infection } & Hong Teng & Sargentgloryvine Stem & Caulis Sargentodoxae \\
\hline & Pu Gong ying & Herba Taraxaci Mongolici & Mongolian Dandelion Herb \\
\hline \multirow[t]{2}{*}{ Increase trace elements } & Nv Zhen Zi & Fructus ligustri lucidi & Ligustrum seed/glossy privet fruit \\
\hline & Xian Mao & Rhizoma Curculiginis & Common Curculigo Rhizome \\
\hline
\end{tabular}

\section{Massage}

The field of massage therapy is a diverse profession made up of a vast array of techniques that share common goals and objectives for providing client/patient-centered health care and wellness that involves human touch(74-77). With its origins in ancient practices from around the world, massage therapy shares common roots with other bodywork and body-centered therapies. At present, some researches utilized massage therapy in male infertility, including body work, foot reflex zone massage, and drug penetration massage.

Ejaculation disorders Spinal cord-injured men with ejaculation disorders can have children following assisted reproduction techniques. Spermatozoa from these patients are usually obtained through vibratory stimulation with or without general anaesthesia, which are expensive and uncomfortable techniques. In a systematic review, there are four articles dealing with prostatic massage in anejaculatory patients (78). 
Prostatic massage appears to be an easy, non-traumatic and risk-free method, as well as a simple outpatient clinic procedure to obtain spermatozoa from paraplegic patients $(79,80)$. Moreover, vigorous prostatic massage was utilized to retrieve spermatozoa from patients with psychogenic anejaculation for ICSI. One case retrieved spermatozoa were successfully used for ICSI(81).

Prospermia is one of the refractory disorders in andrology. Wang et al.(82) in a clinical trial recruited 60 cases of prospermia. In massage series, the pushing, kneading, tapping, pressing, and rubbing manipulations were used and some acupoints of the Ren Channel were strongly stimulated. After once daily for 30 days of treatment, the effect of massage for prospermia was significantly superior to that of acupuncture therapy, especially for functional prospermia and the prospermia caused by chronic prostatitis. Therefore, vigorous prostatic massage could be an effective method of sperm retrieval for assisted conception in patients with ejaculation disorder.

Chronic prostatitis Prostatitis is characterized by voiding symptoms and genitourinary pain and is sometimes associated with sexual dysfunction. Few controlled trials support various non-pharmacological treatments. Chang et al.(83) showed that the mean of TNF- $\alpha$ and IL-8 in prostatic fluid for the patients with chronic nonbacteral prostatitis decreased in the treatment group when compared that of the control group after foot reflex zone massage combining with Qian-lie-tong-yu capsule, and massage is superior to capsule alone treatment. In addition, Meng et al.(84) studied the clinical effect of an innovated computerized pulse water-sac massage with drug penetration instrument (MPWMDPI) on infertility caused by chronic bacterial prostatitis. Ninty-six patients were treated with MPWMDPI. Its main mechanism was administrated herbal drugs through infusing it into urethra, drugs penetrated to prostatic ducts with pulse water-sac massaged 30 times per minute on prostate per anum. The control group included sixty-four patients who were treated with oral antibiotics. Those patients on whom the massage treatment was proved to be effective was $95.8 \%$, which were significantly higher than in the control group. The density and vitality of sperm, seminal plasma Zn and SOD contents were improved.

Immunological infertility The microcomputerized pulse-water-sac massage with drug penetration instrument has also been tried in 181 men with immunological infertility accompanied by chronic seminal vesiculitis (85). Of these cases, $135(74.6 \%)$ were cured, $37(20.4 \%)$ were treated with significant therapeutic effects and $9(5.0 \%)$ with improved effects. The results of AsAb tests became negative in $85.6 \%$ of the patients after treatment, and the pregnancy rate of their wives was $49.1 \%$. No adverse effects were observed in all cases. 
Thus, the massage seems to play some roles in certain male infertility but the underlying mechanism is still unknown. Further large randomized clinical trials are needed.

\section{Tai Chi, Qi Gong and Yoga}

Tai chi and qigong are mind-body practice that originated in China. It combines meditation with slow, gentle, graceful movements, as well as deep breathing and relaxation, to move vital energy throughout the body and has been shown to have a high level of adherence. A study reports that Tai chi has a benefit in people with chronic disease(86). In recent years, some studies show that Tai chi has beneficial effects on immunity and regular Tai chi exercise for 12 weeks significantly promoted both functional mobility and T-cell functions (87-89). Both Tai chi and qigong provide similar health benefits of physical function, quality of life, and immune function (90).

Yoga is an aerobic practice, including breathing techniques, meditation, relaxation, and both gentle and rigorous exercise. Both male and female infertility patients have increased stress level due to the physical and psychological effects of infertility treatments(91), the practice of yoga directly addresses this issue(92). The practice of meditation and relaxation can help increase the clarity of the mind and give patients the patience to undergo the challenge of infertility treatments. Yoga was used as a way to keep peace and stand up to fears of infertility and decrease self-reported symptoms of anxiety in infertility patients (93). Although no studies have been done to evaluate the effects of Tai chi, qi gong or yoga in male infertility, above studies suggest that these approaches may be effective adjunct treatments for male infertility, and some well-designed RCTs in patients of male infertility are needed in the future.

Figure 1. Eastern medical approaches applied to the primary diseases which can cause male infertility at present.

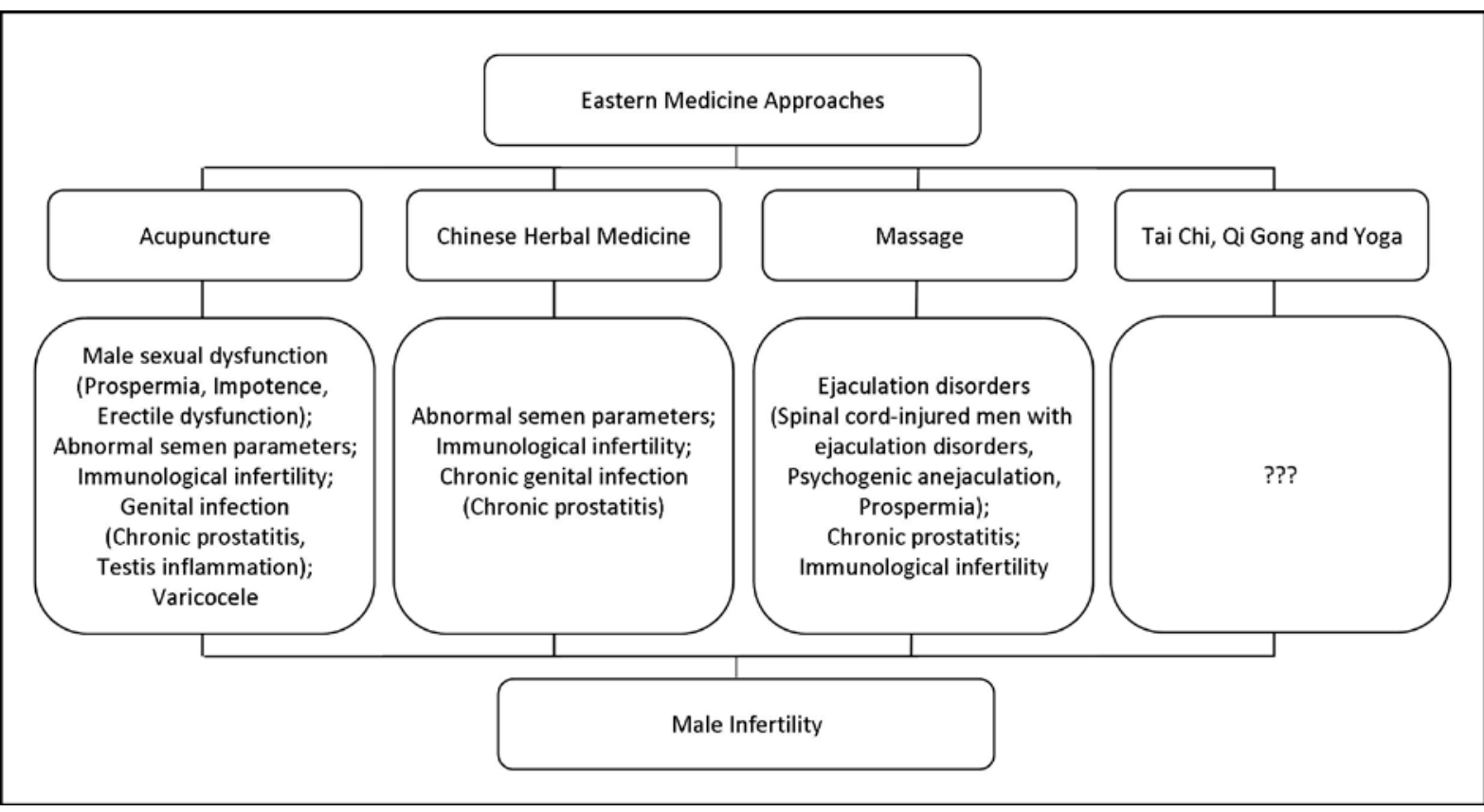




\section{Conclusion}

In conclusion, acupuncture reduces inflammation, increases sperm motility and semen parameters, modulates immune system, as well as improves sexual and ejaculatory dysfunction in male infertility. The clinical effects may mediate via activation of somatic afferent nerves innervating the skin and muscle. Chinese herbal medicines may also exert beneficial effects in male infertility. Unfortunately, most of the studies are case-control reports or just case reports for male infertility, and it is worth noting that some herbal drugs may result in male infertility. Massage also exerts positive effects in male infertility. Nevertheless, the mechanisms of clinical effects have been unclear in male infertility. Tai chi, qi gong and yoga have not been investigated in male infertility, but it has been reported to regulate endocrine and central or autonomic nervous systems involving in male reproduction. Eastern medical approaches may have beneficial on testis blood flow, endocrine, immune system, central and autonomic nervous, and hypothalamic-pituitary-gland, leading to reduction in inflammation, spermatogenesis dysfunction, sexual and ejaculatory disorder. It may be beneficial on reproductive effects in male infertility. However, most studies are small, nonrandomized, or uncontrolled. Therefore, large, well-designed randomized clinical trials are needed to further evaluate the safety, efficacy, and mechanisms of eastern medical approaches for male infertility. 


\section{Reference}

1. World Health Organization. Progress report in reproductive health research, no. 23. Geneva, 2003.

2. Esteves SC, Miyaoka R, Agarwal A. An update on the clinical assessment of the infertile male. Clinics (Sao Paulo). 2011;66(4):691-700.[ PubMed: 21655766]

3. Vincent C, Furnham A. Why do patients turn to complementary medicine? An empirical study. Br J Clin Psychol 1996;35(Pt1):37-48. [PubMed: 8673034]

4. Zini A, Fischer MA, Nam RK, Jarvi K. Use of alternative and hormonal therapies in male infertility. Urology 2004;63:141-3. [PubMed: 14751367]

5. Coulson C, Jenkins J. Complementary and alternative medicine utilisation in NHS and private clinic settings: a United Kingdom survey of 400 infertility patients. J Exp Clin Assist Reprod 2005;2:5. [PubMed: 15807886]

6. Gerhar I, Wallis E. Individualized homeopathic therapy for male infertility. Homeopathy 2002;91: 133-44. [PubMed: 12322866]

7. Smith JF, Eisenberg ML, Millstein SG, Nachtigall RD, Shindel AW, Wing H, Cedars M, Pasch L, Katz PP. The use of complementary and alternative fertility treatment in couples seeking fertility care: data from a prospective cohort in the United States. Fertil Steril. 2010;93(7):2169-2174.[PubMed:20338559]

8. Siterman S, Eltes F, Wolfson V, Zabludovsky N, Bartoov B. Effect of acupuncture on sperm parameters of males suffering from subfertility related to low sperm quality. Arch Androl. 1997:39(2):155-161. [PubMed: 9272232]

9. Ernst E, Posadzki P, Lee MS. Complementary and alternative medicine (CAM) for sexual dysfunction and erectile dysfunction in older men and women: an overview of systematic reviews. Maturitas. 2011;70(1):37-41. [PubMed:21782365]

10. Sunay D, Sunay M, Aydoğmuş Y, Bağbancı S, Arslan H, Karabulut A, Emir L. Acupuncture versus paroxetine for the treatment of premature ejaculation: a randomized, placebo-controlled clinical trial. Eur Urol. 2011;59(5):765-71. [PubMed:21256670]

11. Engelhardt PF, Daha LK, Zils T, Simak R, König K, Pflüger H. Acupuncture in the treatment of psychogenic erectile dysfunction: first results of a prospective randomized placebo-controlled study. Int J Impot Res. 2003;15(5):343-6. [PubMed:14562135]

12. Yaman LS, Kiliç S, Sarica K, Bayar M, Saygin B. The place of acupuncture in the management of psychogenic impotence. Eur Urol. 1994;26(1):52-5. [PubMed:7925530] 
13. Kim KH, Kim TH, Kang JW, Lee MS, Kim JI, Choi JY, Sul JU, Choi SM. Acupuncture for erectile dysfunction in a non-diabetic haemodialysis patient: a case report. Acupunct Med. 2011;29(1):58-60. [PubMed:21169231]

14. Lee MS, Shin BC, Ernst E.Acupuncture for treating erectile dysfunction: a systematic review. BJU Int. 2009;104(3):366-370. [PubMed:19239450]

15. Hirsch A. Male subfertility. BMJ. 2003; 327(7416):669-672. [PubMed:1450443]

16. Franconi G, Manni L, Aloe L, Mazzilli F, Giambalvo Dal Ben G, Lenzi A, Fabbri A. Acupuncture in clinical and experimental reproductive medicine: a review. J Endocrinol Invest. 2011;34(4):307-311. [PubMed:21297382]

17. Siterman S, Eltes F, Schechter L, Maimon Y, Lederman H, Bartoov B. Success of acupuncture treatment in patients with initially low sperm output is associated with a decrease in scrotal skin temperature. Asian J Androl,2009;11(2): 200-208. [PubMed:19122677]

18. Dieterle S, Li CF, Greb R, Bartzsch F, Hatzmann W, Huang DM. A prospective randomized placebo-controlled study of the effect of acupuncture in infertile patients with severe oligoasthenozoospermia. Fertil Steril,2009;92(4):1340-1343. [PubMed:19394002]

19. Zhang M, Huang G, Lu F, Paulus WE, Sterzik K. Influence of acupuncture on idiopathic male infertility in assisted reproductive technology. J Huazhong Univ Sci Technolog Med Sci. 2002;22(3):228-230. [PubMed:12658811]

20. Bidouee F, Shamsa A, Jalali M. Effect of acupuncture on azoospermic male. Saudi J Kidney Dis Transpl,2011;22(5):1039-1040. [PubMed:21912046]

21. Pei J, Strehler E, Noss U, Abt M, Piomboni P, Baccetti B, Sterzik K. Quantitative evaluation of spermatozoa ultrastructure after acupuncture treatment for idiopathic male infertility. Fertil Steril. 2005;84(1):141-147. [PubMed:16009169]

22. Riegler R, Fischl F, Bunzel B, Neumark J. Correlation of psychological changes and spermiogram improvements following acupuncture. Urologe A. 1984;23(6):329-333. [PubMed:6542709]

23. He Xinyun. Acupuncture plus medication for male idiopathic oligospermatic sterility. Shanghai J Acupunct Moxibustion. 1998;2(5):35-37. (Article in Chinese)

20. Gurfinkel E, Cedenho AP, Yamamura Y, Srougi M. Effects of acupuncture and moxa treatment in patients with semen abnormalities. Asian J Androl. 2003;5(4):345-348. [PubMed:14695986]

24. Gao J, Zuo Y, So KKH, Yeung WSB, Ng EHY, Lee KF. Electroacupuncture enhances spermatogenesis in 
rats after scrotal heat- treatment. Spermatogenesis (In press)

26. Lin Wenzhu, Wang Pei. Experimental Acupuncture Science. Shanghai: Shanghai Scientific \& Technical Publishers. 1999,150-152. (Chinese)

27. Fu B,Lun X,Gong Y. Effects of the combined therapy of acupuncture with herbal drugs on male immune infertility. J Tradit Chin Med.2005;25(3):186-9. [PubMed:16334720]

28. Lun X, Rong L. Clinical randomized study on Shu-source point combination for treatment of male immune infertility. Chin Acup \& Moxib.2004;24(3):152-154.(Article in Chinese)

29. Lun X. Effects of electroacupuncture on nitric oxide and trace elements in patient of male immune infertility. Chin Acup \& Moxib.2004;24(12):854-856. (Article in Chinese)

30. Kavoussi B, Ross BE. The neuroimmune basis of anti-inflammatory acupuncture. Integr Cancer Ther. 2007; 6(3):251-257. [PubMed:17761638]

31. Siterman S, Eltes F, Wolfson V, Lederman H, Bartoov B. Does acupuncture treatment affect sperm density in males with very low sperm count? A pilot study. Andrologia. 2000;32(1):31-39. [PubMed:10702864]

32. Wang CY, Han RF. Acupuncture for chronic prostatitis: a meta-analysis. Zhonghua Nan Ke Xue. 2008;14(9):853-856. (Article in Chinese) [PubMed:18998473]

33. Posadzki P, Zhang J, Lee MS, Ernst E. Acupuncture for chronic nonbacterial prostatitis/chronic pelvic pain syndrome: a systematic review. J Androl. 2012;33(1):15-21. [PubMed:21436307]

34. Lee SW, Liong ML, Yuen KH, Leong WS, Chee C, Cheah PY, Choong WP, Wu Y, Khan N, Choong WL, Yap HW, Krieger JN. Acupuncture versus sham acupuncture for chronic prostatitis/chronic pelvic pain.Am J Med. 2008;121(1):79.e1-7. [PubMed:18187077]

35. Ikeuchi T, Iguchi H. Clinical studies on chronic prostatitis and prostatitis-like syndrome (7). Electric acupuncture therapy for intractable cases of chronic prostatitis-like syndrome. Hinyokika Kiyo. 1994;40(7):587-91.(Article in Japanese) [PubMed:8085519]

36. Tugcu V, Tas S, Eren G, Bedirhan B, Karadag S, Tasci A. Effectiveness of acupuncture in patients with category IIIB chronic pelvic pain syndrome: a report of 97 patients. Pain Med. 2010;11(4):518-23. [PubMed:20113410]

37. Lee SH, Lee BC. Electroacupuncture relieves pain in men with chronic prostatitis/chronic pelvic pain syndrome: three-arm randomized trial. Urology. 2009;73(5):1036-41. [PubMed:19394499]

38. Chen R, Nickel JC. Acupuncture ameliorates symptoms in men with chronic prostatitis/chronic pelvic 
pain syndrome. Urology. 2003;61(6):1156-9. [PubMed:12809886]

39. Vital and Health Statistics, series 23, no. 26, CDC. Available from:http://www.cdc.gov

40. Barbieri ER, Hidalgo ME, Venegas A, Smith R, Lissi EA. Varicocele-associated decrease in antioxidant defenses. J Androl. 1999; 20 (6):713-717. [PubMed:105991609]

41. Tarhan S, Gumus B, Gunduz I,AyyildizV, Goktan C. Effect of varicocele on testicular artery blood flow in men-color Doppler investigation. Scand J Urol Nephrol. 2003;37(1):38-42. [PubMed:12745742]

42. Cakmak YO, Akpinar IN, Ekinci G, Bekiroglu N. Point- and frequency-specific response of the testicular artery to abdominal electroacupuncture in humans. Fertil Steril. 2008;90(5):1732-8. [PubMed:18076881]

43. Lee SH, Lee BC. Use of acupuncture as a treatment method for chronic prostatitis/chronic pelvic pain syndromes. Curr Urol Rep. 2011;12(4):288-96. [PubMed:21472420]

44. Lee SW, Liong ML, Yuen KH, Leong WS, Khan NK, Krieger JN. Validation of a sham acupuncture procedure in a randomised, controlled clinical trial of chronic pelvic pain treatment. Acupunct Med. 2011;29(1):40-6. [PubMed:21245238]

45. Raja-Khan N, Stener-Victorin E, Wu X, Legro RS. The physiological basis of complementary and alternative medicines for polycystic ovary syndrome. Am J Physiol Endocrinol Metab. 2011;301(1):E1-E10. [PubMed:21487075]

46. Yang ZX, Chen PD, Yu HB, Pi M, Luo WS, Zhuo YY. Study strategies for acupuncture treatment of chronic nonbacterial prostatitis. Zhong Xi Yi Jie He Xue Bao. 2012;10(3):293-297. [PubMed:22409918]

47. Yuan SY, Qin Z, Liu DS,et al. Acupuncture for chronic pelvic pain syndromes (CPPS) and its effect on cytokines in prostatic fluid. Zhongguo Zhen Jiu. 2011;31(1):11-4.(Article in Chinese) [PubMed:21355145] 48. Zhao DW,Zhao YQ, Jiang SL, Wu CD. Effects of acupuncture on secreted immunoglobulin A in prostatic fluid of patients with chronic nonbacterial prostatitis. Zhongguo Zhen Jiu. 1995;(4):36-37. (Article in Chinese)

49. Lun X. Effects of acutherapy on T lymphocyte subgroup classification in male immuno-infertility. J Beijing University of TCM.2004;27(4):90-92. (Article in Chinese)

50. He Y, Song Y, Zhang X. Therapeutic effect of Guishen pill combined with acupuncture for male immune infertility and influence on acid phosphatase in seminal plasma. Guangzhou University J Tradit Chin Med. 2007; 24(5):359-362. (Article in Chinese)

51. Jiang M, Lu C, Zhang C, Yang J, Tan Y, Lu A, Chan K. Syndrome differentiation in modern research of traditional Chinese medicine. J Ethnopharmacol. 2012;140(3):634-642. Epub 2012 Feb 1. 
[PubMed:22322251]

52. Furuya $\mathrm{Y}$, Akashit T, Fuse H. Effect of BU-ZHONG-YI-QI-TANG on seminal plasma cytokine levels in patients with idiopathic male infertility. Arch Androl. 2004;50(1):11-14. [PubMed:14660164]

53. Amanor T, Hirata A, Namiki M. Effects of Chinese herbal medicine on sperm motility and fluorescence spectra parameters. Arch Androl. 1996;37(3):219-224. [PubMed:8939300]

54. Sun ZM, Bao YZ. TCM treatment of male immune infertility- a report of 100 cases. J Tradit Chin Med. 2006;26(1): 36-38. [PubMed:16705853]

55. Lu TK, O YANG HG, Jin GY. et al. Clinical study on the treatment of male immune infertility by Hu-zhang-dan-shen-yin. National J Androl.2006; 12(8):750-755.

56. Yang BC, Zhang CX, Du L, Shan X, Zou P, Dang Q. Clinical Study on the Treatment of Male Immune Infertility with Sheng-Jing-Zhong-Zi-Tang. J Tradit Chin Med. 2002;22(2): 102-103. [PubMed:12125479] 57. Jia YS, Li YQ, Li J, Sun M. Treatment of nonspecific chronic prostatitis with Qian-Lie-Xian-Yan suppository in 104 cases. J Tradit Chin Med. 2001;21(2): 90-92. [PubMed:11498910]

58. Sun J, Zhou AF, Ding CF. Clincal observation of qing-re-yu-lin decoction intreatment of male infertility caused by accessory gland infection. Zhongguo Zhong Xi Yi Jie He Za Zhi. 2006;26(10):877-880. (Article in Chinese) [PubMed:17121035]

59. Wang WH. What Kinds of External Therapies Can Be Adopted for Chronic Prostatitis? J Tradit Chin Med. 2007; 27(3): 238-240. [PubMed:17955666]

60. Cheong Y, Nardo LG, Rutherford T, Ledger W. Acupuncture and herbal medicine in in vitro fertilisation: a review of the evidence for clinical practice. Hum Fertil (Camb). 2010; 13(1): 3-12. [PubMed:20053149] 61. Qian SZ, Zhong CQ,Xu Y. Effect of Tripterygium wilfordh Hook. f. on the fertility of rats. Contraception. 1986;33(2): 105-110.

62. Qian SZ, Hu YZ, Wang SM, Luo Y, Tang AS, Shu SY, Zhou JW, Rao TY. Effects of Tripterygium hypoglaucum Hutch on male fertility. Adv Contracept.1988; 4(4):307-310. [PubMed:3252728]

63. Matlin SA, Belenguer A, Stacey VE, Qian SZ, Xu Y, Zhang JW, Sanders JK, Amor SR, Pearce CM. Male antifertility compounds from tripterygium wilfordh hook F. Contraception. 1993; 47(4):387-400. [PubMed:8508668]

64. Tempest HG, Homa ST, Routledge EJ, Garner A, Zhai XP, Griffin DK. Plants used in chinese medicine for the treatment of male infertility possess antioxidant and anti-oestrogenic activity. Syst Biol Reprod Med . 2008; 54(4-5):185-195. [PubMed:18942026] 
65. Wang XM, Fu H, Liu GX. Clinical and experimental study on effect of Wu-Zi-Yan-Zong pill on oxidative damage of mitochondrial DNA in aging. Chin J Integr Tradit West Med. 2002, 22(2):101. (Article in Chinese)

66. Chen KH, Fang J, Kuang XW. The effect of extracting solution of Raspberry on hypothalamic-pituitary-gonadal axis function. Chin J Chin Mater Med. 1996;21(9):560-562. (Article in Chinese)

67. Chang MS, Kim WN, Yang WM, Kim HY, Oh JH, Park SK. Cytoprotective effects of Morinda officinalis against hydrogen peroxide-induced oxidative stress in Leydig TM3 cells. Asian J Androl. 2008; 10(4):667-674. [PubMed:18478165]

68. Peng SJ, Lu RK,Yu LH. Effects of semen Cuscutae, rhizoma Curculiginis, radix Morindae officinalis on human spermatozoan's motility and membrane function in vitro. Zhongguo Zhong Xi Yi Jie He Za Zhi. 1997; 17(3):145-147. (Article in Chinese) [PubMed:9863078]

69. Aitken RJ, Baker MA. Oxidative stress, sperm survival and fertility control. Mol Cell Endocrinol. 2006; 250(1-2):66-69. [PubMed:16412557]

70. Qin ND, She YC. The effect of $\mathrm{Tu} \mathrm{Si} \mathrm{Zi}$ and the main compounds flavonoids on hypothalamic-pituitary-gonadal axis function. J shanton university medical college. 1998; 11(3):84-85. (Article in Chinese)

71. Xuejiang W, Ichikawa H, Konishi T. Antioxidant potential of qizhu tang, a chinese herbal medicine, and the effect on cerebral oxidative damage after ischemia reperfusion in rats. Biol Pharm Bull 2001; 24(5):558-563. [PubMed:11379780]

72. Wang Q. TCM reproduction medicine history, eurrent situation and prospect. Chin J Hum Sexuality. 2005;14(4):3-7. (Article in Chinese)

73. Dai N. The mechanism research of traditional Chinese medicine for the male infertility. Reproduction \& contraception 1997;17(4):200-203. (Article in Chinese)

74. Hertenstein MR, Holmes M, McCullough M, Keltner D. The communication of emotion via touch. Emotion. 2009;9(4):566-573. [PubMed:19653781]

75. Immordino-Yang MH, McColl A, Damasio H, Damasio A. Neural correlates of admiration and compassion. Proc Natl Acad Sci U S A. 2009;106(19):8021-8026. [PubMed:19414310]

76. Cherkin DC, Sherman KJ, Kahn J, Erro JH, Deyo RA, Haneuse SJ, Cook AJ.Effectiveness of focused structural massage and relaxation massage for chronic low back pain: protocol for a randomized 
controlled trial. Trials. 2009;10:96. [PubMed:19843340]

77. Sherman KJ, Cherkin DC, Kahn J, Erro J, Hrbek A, Deyo RA, Eisenberg DM. A survey of training and practice patterns of massage therapists in two US states. BMC Complement Altern Med. 2005;5:13. [PubMed:15955245]

78. Kamischke A, Nieschlag E. Update on medical treatment of ejaculatory disorders. Int J Androl. 2002;25(6):333-44. [PubMed:12406365]

79. Marina S, Marina F, Alcolea R, Nadal J, Pons MC, Grossmann M,Rosabel E, Vidal J. Triplet pregnancy achieved through intracytoplasmic sperm injection with spermatozoa obtained by prostatic massage of a paraplegic patient. Hum Reprod, 1999;14(6):1546-1548. [PubMed:10357973]

80. Arafa MM, Zohdy WA, Shamloul R. Prostatic massage: a simple method of semen retrieval in men with spinal cord injury. Int J Androl. 2007;30(3):170-3. [PubMed:17298549]

81. Fahmy I, Kamal A, Metwali M, Rhodes C, Mansour R, Serour G, Aboulghar M. Vigorous prostatic massage: a simple method to retrieve spermatozoa for intracytoplasmic sperm injection in psychogenic anejaculation: case report. Hum Reprod. 1999;14(8):2050-3. [PubMed:10438425]

82. Wang C, Shi J. Therapeutic effect of massotherapy for prospermia. J Tradit Chin Med.2000;20(2):136-7. [PubMed:11039006]

83. Chang $\mathrm{D}, \mathrm{Li} \mathrm{G}$,Zhang P,Wu T,Zhang C. Foot reflex zone massage type of blood stasis in patients with chronic abacterial prostatitis inprostatic fluid of TNF- $\alpha$,IL-8. Liaoning J Tradit Chin Med. 2011;38(5):804-7. (Article in Chinese)

84. Meng Z, Luo E. Treating chronic bacterial prostatitis caused infertility with microcomputerized pulse water-sac massage drug penetration instrument. Reproduction \& Contraception.2003;23(2):84-7. (Article in Chinese)

85. Meng Z, Luo E. A microcomputerized pulsed water-sac massage with drug penetration instrument for treatment of male patients with immunity infertility accompanied by chronic seminal vesiculitis. Sheng Wu Yi Xue Gong Cheng Xue Za Zhi. 2003;20(3):460-2. (Article in Chinese) [PubMed:14565013]

86. Wang C, Collet JP, Lau J. The effect of tai chi on health outcomes in patients with chronic conditions. Arch Intern Med 2004;164:493-501. [PubMed:15006825]

87. Yeh SH, Chuang H, Lin LW, Hsiao CY, Eng HL. Regular tai chi chuan exercise enhances functional mobility and CD4CD25 regulatory T cells. Br J Sports Med 2006;40:239-43. [PubMed:16505081] 88. Yeh SH, Chuang H, Lin LW, Hsiao CY, Wang PW, Yang KD. Tai chi chuan exercise decreases HbA1c level 
along with increase of regulatory T-cells and decrease of cytotoxic T-cell population in type 2 diabetic patients. Diabetes Care 2007;30(3):716-718. [PubMed:17327347]

89. Yeh SH, Chuang H, Lin LW, Hsiao CY, Wang PW, Liu RT, Yang KD. Regular Tai Chi Chuan exercise improves $\mathrm{T}$ cell helper function of patients with type 2 diabetes mellitus with an increase in T-bet transcription factor and IL-12 production. Br J Sports Med 2009;43(11): 845-850. [PubMed:18385192]

90. Jahnke R, Larkey L, Rogers C, Etnier J, Lin F. A comprehensive review of health benefits of qigong and tai chi. Am J Health Promot, 2010;24(6):e1-e25. [PubMed:20594090]

91. Khalsa HK. Yoga: an adjunct to infertility treatment. Fertil Steril. 2003 Oct;80 Suppl 4:46-51.[PubMed:14568288]

92. West J, Otte C, Geher K, Johnson J, Mohr DC. Effects of Hatha yoga and African dance on perceived stress, affect, and salivary cortisol. Ann Behav Med 2004;28:114-18. [PubMed: 15454358]

93. Galantino ML, Bzdewka TM, Eissler-Russo JL, Holbrook ML, Mogck EP, Geigle P, et al. The impact of modified Hatha yoga on chronic low back pain: a pilot study. Altern Ther Health Med 2004;10:56-59. [PubMed: 15055095] 